\title{
Die einfache Extraktion und Quantifizierung von sprengstofftypischen Verbindungen in Bodenproben über LC-MS/MS
}

\author{
Liane Kober, Christian Hanschke, Rainer Macholz, Marcus Frohme*
}

\section{Zusammenfassung}

Die Kontamination von Flächen mit Rüstungsaltlasten durch sprengstofftypische Verbindungen (STV) wie 2,4,6-Trinitrotoluol (TNT) ist nach wie vor ein großes Risiko für Gesundheit und Umwelt. Die Hochdruckflüssigchromatographie (HPLC) ist in Kombination mit der Tandem-Massenspektrometrie (MS/MS) ein besonders sensitives und exaktes Verfahren zur Konzentrationsbestimmung derartiger Umweltkontaminanten. Jedoch benötigen die derzeit eingesetzten Methoden zur Extraktion und Quantifizierung viel Zeit und Bodenmaterial. Wir haben daher eine 20-minütige LC-MS/MS-Methode entwickelt, die in einer Probe simultan acht STV qualitativ und quantitativ nachweisen kann. Im Vergleich zur EPA-Referenzmethode konnte bei der Extraktion die eingesetzte Bodenmenge von $10 \mathrm{~g}$ auf $2 \mathrm{~g}$ reduziert und die Extraktionsdauer von $18 \mathrm{~h}$ auf 30 min verkürzt werden. Die abschließende Validierung zeigte eine gute Reproduzierbarkeit und mit etablierten Protokollen vergleichbare Detektions- (LOD) und Quantifizierungslimits (LOQ), so dass die optimierten Methoden für eine schnelle und zuverlässige Analyse von Realproben eingesetzt werden konnten.

\section{Abstract}

Soil contamination on former military sites with explosives-related compounds (STV) such as 2,4,6-trinitrotoluene (TNT) is still a major health and environmental threat. High pressure liquid chromatography (HPLC) combined with tandem mass spectrometry (MS/MS) is a very sensitive and precise method to detect such environmental contaminants. However, currently used extraction and quantification methods often need a long time and a high sample amount. Thus, we developed an LC-MS/MS method that detects and quantifies eight explosives simultaneously in only $20 \mathrm{~min}$. Compared to the EPA reference method, the amount of soil was reduced from $10 \mathrm{~g}$ to $2 \mathrm{~g}$ and the extraction time shortened from $18 \mathrm{~h}$ to $30 \mathrm{~min}$. The validation revealed a good reproducibility of the fast analysis process. Limits of detection (LOD) and quantification (LOQ) were comparable to established protocols. Hence, the optimized methods were used for a fast and reliable quantification of STV in soil samples of a former military site.

\section{Einleitung}

Auf Altlastenverdachtsflächen werden immer noch großflächige Kontaminationen mit Sprengstoffen gefunden. Allein im Land Brandenburg gelten 400.000 ha als belastet (Küchler 2011). Die Stoffe sind nicht nur durch ihre Explosivkraft, sondern auch durch ihre hohe Toxizität ein erhebliches Umweltproblem (Herrmann 2008). Insbesondere TNT wurde in den zurückliegenden zwei Weltkriegen in großen Mengen produziert. Durch Transformationsreaktionen entstehen verschiedene
TNT-Abbauprodukte, die neben dem Boden auch das Grundwasser belasten. Mit den Neben- und Zwischenprodukten sowie weiteren als Sprengstoff genutzten Nitroverbindungen wie z. B. 1,3,5-Trinitrobenzol (TNB) bilden sie eine Vielzahl an Verbindungen, die unter dem Begriff der sprengstofftypischen Verbindungen (STV) zusammengefasst werden (Landesamt für Umwelt, Gesundheit und Verbraucherschutz Brandenburg 2015).

Die Verteilung der STV im Boden ist aufgrund ihres Ursprungs als hochkonzentrierte Aggregate nicht homogen. In Abhängigkeit von der Her- kunft, der Bodenbeschaffenheit und den Umwelteinflüssen verändern sich ihre Abbauraten und lokalen Konzentrationen über die Zeit. Insbesondere humushaltiger Boden weist eine hohe Kapazität auf, große Mengen STV adsorptiv zu binden (Via \& Zinnert 2016). Unter anaeroben Bedingungen wird nicht adsorbiertes TNT oft schnell in seine Abbauprodukte umgewandelt (Environmental Protection Agency 2017). Neben abiotischen Transformationen durch Licht, Metallionen oder ein alkalisches Milieu können die STV auch durch Mikroorganismen transformiert werden. Unter an- 
aeroben Bedingungen findet häufig eine Reduktion der Nitrogruppen zu Aminogruppen statt, die nicht weiter abgebaut werden können und in der Umwelt verbleiben (Monteil-Rivera et al. 2009). Beim Abbau von TNT werden so unter anderem Aminodinitrotoluole (ADNT) gebildet, die nach TNT als zweithäufigste Kontamination auf Altlastenverdachtsflächen gefunden werden können.

Die Intoxikation mit TNT durch Inhalation oder Hautkontakt wurde insbesondere für die an der Herstellung von Explosivstoffen beteiligten Arbeiter beschrieben, sie kann aber auch durch den Kontakt mit kontaminierten Böden oder hoch belastetem Grundwasser erfolgen. Studien legen nahe, dass die Toxizität von TNT und seinen Abbauprodukten auf die Generierung von reaktiven Sauerstoffverbindungen zurückzuführen ist, die die Leber, das Blut, das Immunsystem und die Reproduktionsfähigkeit schädigen können (Environmental Protection Agency 2017).

Um das Ausmaß der STV-Ausbreitung und das damit verbundene Gefährdungspotential einschätzen zu können, werden daher genaue und schnelle Analysemethoden benötigt, die die Probenaufarbeitung des Bodens, die Extraktion der STV aus dem Boden und die Ermittlung der STV-Konzentration mit geeigneten Methoden einschließt. Letzteres kann im einfachsten Fall über eine chromatographische Auftrennung und UV/Vis-Detektion bei $254 \mathrm{~nm}$ durchgeführt werden. Zur Erhöhung der Sensitivität und Selektivität kann die Chromatographie mit einem Massenspektrometer gekoppelt werden, wobei lonen der STV gemäß ihrem Masse-Ladungs-Verhältnis $(\mathrm{m} / \mathrm{z})$ detektiert werden (Landesamt für Umwelt, Gesundheit und Verbraucherschutz Brandenburg 2015). Bei der Triple-Quadrupol-Technologie können zudem zunächst Produkt-Ionen selektiert, in einer Kollisionszelle fragmentiert und schließlich spezifische Fragment-Ionen der STV nachgewiesen werden. Dadurch wird die Spezifität und Sensitivität der Methode weiter erhöht und eine sehr genaue Quantifizierung der im Boden enthaltenen STV ermöglicht.
Neben der Analysemethode kommt der Probenvorbereitung eine besondere Bedeutung zu. Aufgrund der inhomogenen Verteilung der STV im Boden ist die Homogenisierung der Proben ein wichtiger Bestandteil, um reproduzierbare und richtige Ergebnisse zu erhalten. Im Anschluss daran kann in einem einfachen Extraktionsverfahren die Bodenprobe mit Lösungsmittel versetzt und über einen längeren Zeitraum (ca. 18 h) geschüttelt werden (Deutsches Institut für Normung 2014; Environmental Protection Agency 2006). Die im Boden enthaltenen STV lösen sich dabei im organischen Lösungsmittel und können nach Filtration am LC-MS/MS quantifiziert werden.

Um eine gute Auftrennung der strukturell sehr ähnlichen STV zu erreichen und gleichzeitig eine zeitsparende Analyse zu etablieren, soll eine LC-MS/ MS-Methode zum simultanen Nachweis von acht relevanten STV entwickelt werden. Für die untersuchten STV wird zudem überprüft, ob die Extraktion in Anbetracht bestehender Referenzmethoden vereinfacht und beschleunigt werden kann. Eine abschließende Validierung der Methoden und Testung an Realproben soll schließlich für die Bewertung herangezogen werden, ob diese in Zukunft für eine schnellere und flächendeckende Analyse belasteter Altlastenverdachtsflächen genutzt werden können.

\section{Material und Methoden}

\subsection{Chemikalien}

Alle verwendeten Chemikalien und Lösungsmittel wiesen den Reinheitsgrad p.A., HPLC oder LC-MS auf und wurden von der Carl Roth $\mathrm{GmbH}$ (Karlsruhe) erworben. Folgende STVStandards wurden von der SigmaAldrich Chemie $\mathrm{GmbH}$ (Taufkirchen) bezogen: 2-Amino-4,6-Dinitrotoluol (2-ADNT), 4-Amino-2,6-Dinitrotoluol (4-ADNT), 1,3-Dinitrobenzol (DNB), 2-Nitrotoluol (2-NT) und 1,3,5-Trinitrobenzol (TNB). 2,4,6-Trinitrotoluol (TNT), 2,4-Dinitrotoluol (2,4-DNT) und 2,6-Dinitrotoluol (2,6-DNT) sowie die belasteten Bodenproben wurden von der terracon $\mathrm{GmbH}$ (Jüterbog) zur Verfügung gestellt.
2.2 Herstellung von Standardlösungen

Für die Methodenentwicklung wurden Stammlösungen der acht STVStandards (TNT, 2-ADNT, 4-ADNT, TNB, DNB, 2,4-DNT, 2,6-DNT, 2-NT) sowie ein Gemisch mit allen STV mit Konzentrationen von $1 \mu \mathrm{g} / \mathrm{ml}$ und $10 \mu \mathrm{g} / \mathrm{ml}$ in Acetonitril erstellt. Für die Kalibration wurden Konzentrationen von $0,01 \mu \mathrm{g} / \mathrm{ml}$ bis $50 \mu \mathrm{g} / \mathrm{ml}$ des STVGemischs (0,01; 0,025; 0,05; 0,10; 0,25; $0,5 ; 1,0 ; 5,0 ; 10,0 ; 25,0 ; 50,0 \mu \mathrm{g} / \mathrm{ml})$ verwendet.

\subsection{Vorbereitung der Bodenproben}

Zur Bereitstellung einer definierten Bodenbelastung wurden Sand- und Humusboden mit TNT dotiert. Hierzu wurden unbelasteter Sand bzw. Humus getrocknet, auf $2 \mathrm{~mm}$ Partikelgröße gesiebt, mit TNT versetzt, $10 \mathrm{~min}$ gemischt, um dann erneut gesiebt und für 10 min gemischt zu werden. Die Endkonzentrationen betrugen für beide Bodenarten $1 \mathrm{~g} \mathrm{TNT} / \mathrm{kg}$ Boden. Die von der terracon $\mathrm{GmbH}$ zur Verfügung gestellten Bodenproben wurden vor der Entnahme der Stichproben gemischt und der Wassergehalt der Proben wurde zur Berücksichtigung in der Konzentrationsberechnung bestimmt.

\subsection{STV-Extraktion aus Bodenproben}

Die Extraktion wurde gemäß der von der EPA beschriebenen Methode 8330b durchgeführt (Environmental Protection Agency 2006). Kurz zusammengefasst wurden die Bodenproben im Dunkeln getrocknet, auf $2 \mathrm{~mm}$ Partikelgröße gesiebt, $10 \mathrm{~g}$ abgewogen und in $20 \mathrm{ml}$ Acetonitril auf dem Schüttler (Intelli Mixer RM-21, Elmi) für $18 \mathrm{~h}$ extrahiert. Zur Vereinfachung der Extraktion wurden verschiedene Experimente mit den dotierten Bodenproben durchgeführt. Hierzu wurden die Verkürzung der Extraktionszeit (1 min, $5 \mathrm{~min}, 10 \mathrm{~min}, 30 \mathrm{~min}, 60 \mathrm{~min}, 18 \mathrm{~h}$ ) sowie die Verringerung des Bodenmaterials $(1 \mathrm{~g}, 2 \mathrm{~g}, 5 \mathrm{~g}, 10 \mathrm{~g}$ ) bei gleichbleibendem Verhältnis zum Lösungsmittel Acetonitril untersucht. Als Alternative wurde zudem eine Ultraschallextraktion mit den o. g. Zeiten betrachtet. Da zur Kalibration Standardlösungen verwendet wurden, erfolgte eine Va- 
lidierung der Methoden mit dotierten Extrakten. Zu diesem Zweck wurden die Wiederfindungsrate $(10 \mu \mathrm{g} / \mathrm{ml} \mathrm{STV-}$ Gemisch) und die Präzision ( $n=10)$ bei Anwendung der optimierten Methoden bestimmt sowie Realproben analysiert.

Vor der Messung an der LC-MS/MS wurden alle Extrakte zentrifugiert (2 min, 10.000xg), der Überstand filtriert (PTFE, 0,22 $\mu \mathrm{m}$ ) und im Verhältnis 1:10 bzw. 1:100 mit Acetonitril verdünnt.

\subsection{Chromatographie und MS-Optimierung}

Die LC-MS/MS-Analysen wurden am LCMS-8040 (Shimadzu Europa GmbH, Duisburg), bestehend aus einer HPLC mit Photodiodenarray (PDA) und einem Triple-Quadrupol-Massenspektrometer mit Elektrospray-Ionisierung (ESI), durchgeführt. Für die Gerätesteuerung und Auswertung der Ergebnisse wurde die Software LabSolutions (Shimadzu) verwendet. Die Auftrennung der STV erfolgte mithilfe der HPLC-Säule Nucleodur ${ }^{\circledR} \mathrm{C} 18$ HTec, $3 \mu \mathrm{m}, 2 \times 150$ mm (MachereyNagel GmbH \& Co. KG, Düren) und dem zugehörigen Vorsäulensystem (Column Protection System, Macherey-Nagel). Als mobile Phase wurde ein Gradient aus Wasser mit 5 mM Ammoniumacetat und 0,001 \% Ammoniumhydroxid (A) sowie Methanol (B) verwendet. Eine optimale Auftrennung konnte bei einer Säulentemperatur von $28^{\circ} \mathrm{C}$, einer Flussrate von $0,15 \mathrm{ml} / \mathrm{min}$ und dem in Tab. 1 dargestellten Gradienten erreicht werden. Die UV-Detektion und -Quantifizierung erfolgte bei $254 \mathrm{~nm}$. Das Injektionsvolumen betrug $1 \mu \mathrm{l}$.

Im Massenspektrometer konnten im negativen lonenscan fünf der acht STV anhand spezifischer ProduktIonen identifiziert werden. Über das Multiple-Reaction-Monitoring (MRM) wurden für diese fünf STV die häufigsten Fragmente bestimmt (Tab. 2). Mit Ausnahme der ADNT wurde bei allen STV das häufigste Fragment $\left(^{*}\right)$ zur Quantifizierung herangezogen. Die optimierten Bedingungen waren hierfür eine ESI-Kapillarspannung von $-3,5 \mathrm{kV}$, eine lonenquellentemperatur von $250^{\circ} \mathrm{C}$ und eine Heizblocktemperatur von $400^{\circ} \mathrm{C}$. Als Verneblungsund Trocknungsgas wurde Stickstoff

Tab. 1: Profil des Lösungsmittelgradienten zur STV-Separation

\begin{tabular}{|l|l|l|}
\hline $\begin{array}{l}\text { Zeit } \\
{[\mathrm{min}]}\end{array}$ & $\begin{array}{l}\text { Lösungsmittel A } \\
{[\%, \mathrm{v} / \mathrm{v}]}\end{array}$ & $\begin{array}{l}\text { Lösungsmittel B } \\
{[\%, \mathrm{v} / \mathrm{v}]}\end{array}$ \\
\hline 0,0 & 43 & 57 \\
\hline 2,0 & 43 & 57 \\
\hline 13,0 & 30 & 70 \\
\hline 14,8 & 30 & 70 \\
\hline 15,0 & 90 & 10 \\
\hline 20,0 & 90 & 10 \\
\hline
\end{tabular}

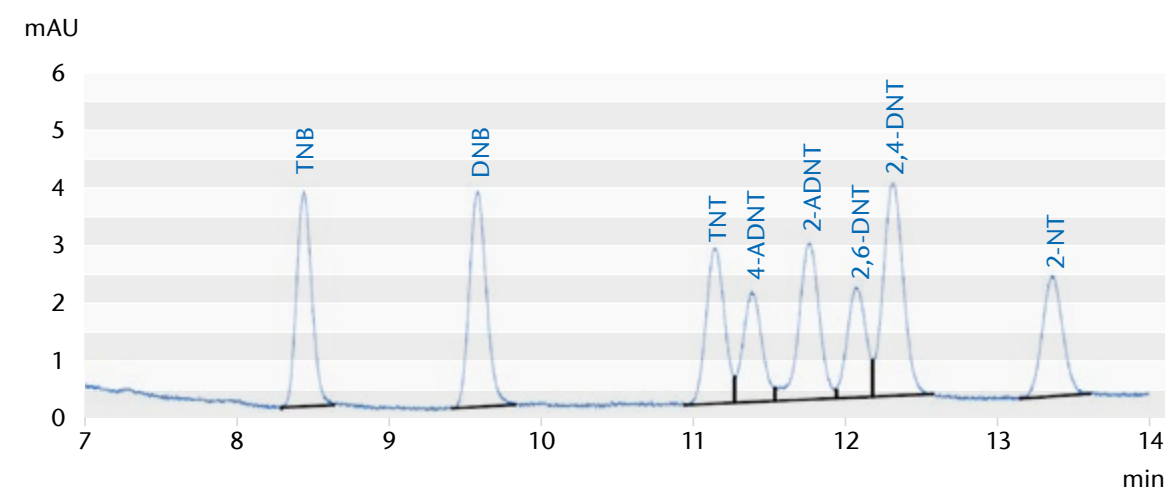

Abb. 1) Chromatographische Auftrennung und UV-Detektion (254 nm) eines 1- $\mu \mathrm{g} / \mathrm{ml}$-STV-Standardgemischs mit TNB, DNB, TNT, 4-ADNT, 2-ADNT, 2,6-DNT, 2,4-DNT und 2-NT.

eines Stickstoffgenerators (Infinity NM32L, Peak Scientific Instruments $\mathrm{GmbH}$, Düren) mit einer Flussrate von 3 bzw. 15 l/min eingesetzt. Das Gas Argon wurde zur Fragmentierung der STV in der Kollisionszelle genutzt (230 kP).

\section{Ergebnisse}

\subsection{LC-MS/MS-Methodenentwicklung}

Im Zuge der chromatographischen Methodenentwicklung konnten die acht strukturell sehr ähnlichen STV erfolgreich separiert werden (Abb. 1). Insbesondere für die Trennung der Isomere 4- und 2-ADNT bzw. 2,4- und 2,6-DNT spielten eine genaue Einstellung des Gradienten (Tab. 1) sowie die Säulentemperatur $\left(28^{\circ} \mathrm{C}\right)$ eine wichtige Rolle.

Im negativen lonenscan des Massenspektrometers konnten für fünf der acht Verbindungen spezifische lonen detektiert werden. Für TNT, 4-ADNT, 2-ADNT und 2,4-DNT wurde das de- protonierte Anion [M-H]- nachgewiesen. Bei TNB zeigte sich dagegen eine größere Abweichung zum Molekulargewicht (s. Tab. 2). Für die ionisierbaren STV wurden in der automatisierten MRM-Optimierung die zwei bis fünf häufigsten Fragmente bestimmt, von denen in den meisten Fällen das häufigste zur Quantifizierung genutzt wurde. Die weiteren Fragmente dienten zur Qualitätssicherung. Bei 2-ADNT und 4-ADNT wurde bei der Auswahl des Fragments zur Quantifizierung berücksichtigt, dass das Fragment beim jeweils anderen Isomer ein möglichst geringes Signal hervorruft. Aufgrund der sehr ähnlichen Struktur und des daraus resultierenden Fragmentierungsverhaltens ist in den MRM-Chromatogrammen zu den Elutionszeiten jeweils trotzdem ein Doppelpeak zu erkennen (Abb. 2). Daher ist insbesondere für die ADNT nicht nur die Auswahl spezifischer Fragmente, sondern auch der Elutionszeitpunkt ein wichtiger Faktor, um den korrekten Peak zur Quantifizierung heranzuzie- 


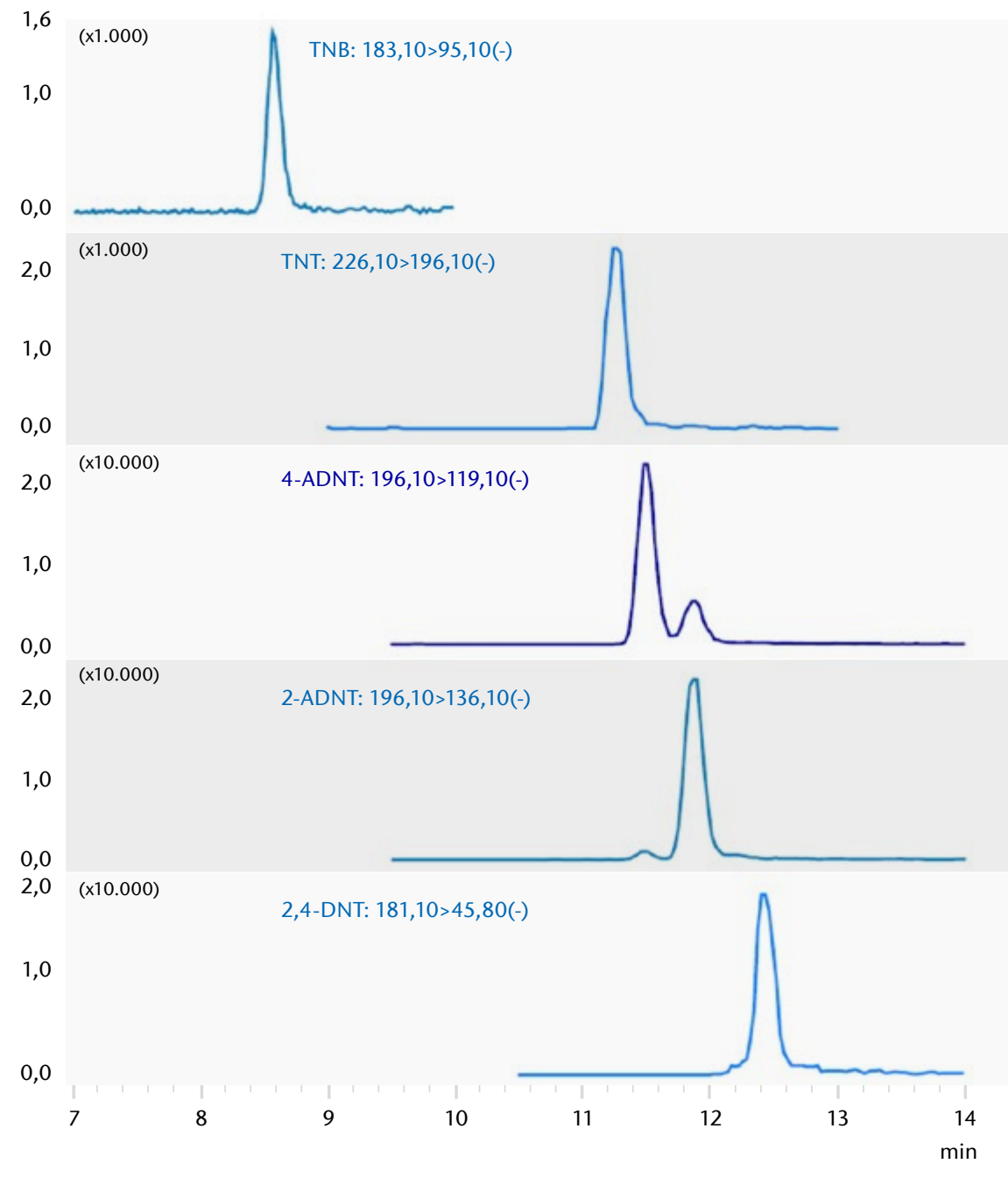

Abb. 2) MRM-Chromatogramme der fünf über ESI ionisierbaren STV TNB, TNT, 4-ADNT, 2-ADNT und 2,4-DNT eines 1- $\mu \mathrm{g} / \mathrm{ml}$-STV-Standardgemischs mit Kennzeichnung und Benennung der zur Quantifizierung genutzten Fragmente. hen. Im Vergleich zu den anderen drei STV ist der Nachweis der Aminodinitrotoluole um den Faktor 10 sensitiver.

\subsection{Validierung der LC-MS/MS- Methoden}

Zur Konzentrationsbestimmung in Bodenproben wurden Kalibrationsgeraden für die STV mithilfe der UVChromatogramme und der MRMChromatogramme erstellt. In beiden Detektionsverfahren zeigt die Mehrfachbestimmung nur eine geringe Standardabweichung von durchschnittlich $5 \%(0,17-16,6 \%)$ mit einem Bestimmtheitsmaß $\mathrm{R}^{2}$ von 0,999 bis 1. Die massenspektrometrische Quantifizierung weist gegenüber der UV-Quantifizierung eine größere Sensitivität auf (vgl. Abb. 3 für 4-ADNT). Daher erstreckt sich auch der lineare Bereich in einem niedrigeren Konzentrationsbereich als die UV-Kalibration (Tab. 3).

Zur Bestimmung der Nachweis- (LOD) und Quantifizierungslimits (LOQ) wurde das Signal-Rausch-Verhältnis $(\mathrm{S} / \mathrm{N})$ der Peaks herangezogen. Ein S/N $>3$ wurde als untere Nachweis-, ein $\mathrm{S} / \mathrm{N}>10$ als Quantifizierungsgrenze festgelegt (DeArmond \& DiGoregorio 2013; Leito et al. 2017). Die massenspektrometrische Detektion ist auch hier bis zu 25-mal sensitiver (Tab. 3).

Tab. 2: Molekulargewicht der untersuchten STV, Elutionszeitpunkte im UV-Chromatogramm (254 nm), Nachweisbarkeit im MS (ESI) sowie spezifische Produkt-Ionen und Fragment-Ionen mit Kennzeichnung des für die Quantifizierung genutzten Fragments $\left({ }^{*}\right)$.

\begin{tabular}{|c|c|c|c|c|c|}
\hline $\begin{array}{l}\text { STV } \\
{[-]}\end{array}$ & $\begin{array}{l}\mathrm{M}_{\mathrm{w}} \\
{[\mathrm{g} / \mathrm{mol}]}\end{array}$ & $\begin{array}{l}\text { UV: } 254 \mathrm{~nm} \\
\text { [min] }\end{array}$ & $\begin{array}{l}\text { MS: ESI- } \\
{[-]}\end{array}$ & $\begin{array}{l}\text { Produkt-Ion } \\
{[\mathrm{m} / \mathrm{z}]}\end{array}$ & $\begin{array}{l}\text { Fragment-Ionen } \\
{[\mathrm{m} / \mathrm{z}]}\end{array}$ \\
\hline TNT & 227,1 & 10,9 & $\checkmark$ & 226,1 & $196,10 * ; 46,00 ; 64,05$ \\
\hline 4-ADNT & 197,1 & 11,2 & $\checkmark$ & 196,1 & 166,$30 ; 149,15 ; 136,10 ; 119,15^{*} ; 46,00$ \\
\hline 2-ADNT & 197,1 & 11,6 & $\checkmark$ & 196,1 & 166,$10 ; 150,10 ; 136,10 * ; 119,15 ; 45,85$ \\
\hline 2,6-DNT & 182,1 & 11,9 & - & - & - \\
\hline 2,4-DNT & 182,1 & 12,2 & $\checkmark$ & 181,1 & 135,$25 ; 116,05 ; 105,20 ; 45,80^{*}$ \\
\hline 2-NT & 137,1 & 13,1 & - & - & - \\
\hline TNB & 213,1 & 8,3 & $\checkmark$ & 183,1 & 125,$10 ; 95,10^{*}$ \\
\hline DNB & 168,1 & 9,4 & - & - & - \\
\hline
\end{tabular}


Um die Selektivität der chromatographischen Auftrennung zu bewerten, wird die Peakauflösung $R_{s}$ genutzt. Sie ist ein Maß dafür, wie weit benachbarte Peaks (A und B) im Chromatogramm auseinanderliegen. Für die Berechnung werden die Retentionszeiten $t_{R}$ und die Halbwertsbreiten $w_{1 / 2}$ der Peaks benötigt.

$$
R_{S}=\frac{t_{R}(B)-t_{R}(A)}{0,85\left(w_{1 / 2}(B)-w_{1 / 2}(A)\right)}
$$

Als Richtwert kann hier eine Auflösung von mindestens 1,5 herangezogen werden (Leito et al. 2017). Dieser Wert wurde für die meisten STV erreicht (Tab. 3). Lediglich TNT und 4-ADNT $(1,13), 2$-ADNT und 2,4 -DNT $(0,98)$ sowie 2,4-DNT und 2,6-DNT $(0,96)$ zei- gen eine schlechtere Peakauflösung. Beim MRM-basierten Nachweis wird dieser Parameter durch die spezifische Produkt- und Fragment-Ion-Selektion nicht benötigt. Neben der hohen Sensitivität ist diese Selektivität ein weiterer Grund, zur Bestimmung der STV-Konzentrationen die MRM-Quantifizierung der UV-Quantifizierung vorzuziehen.

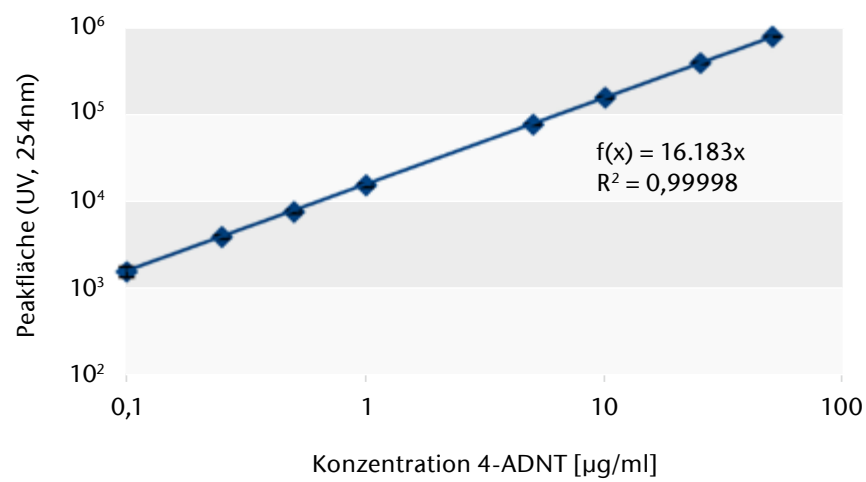

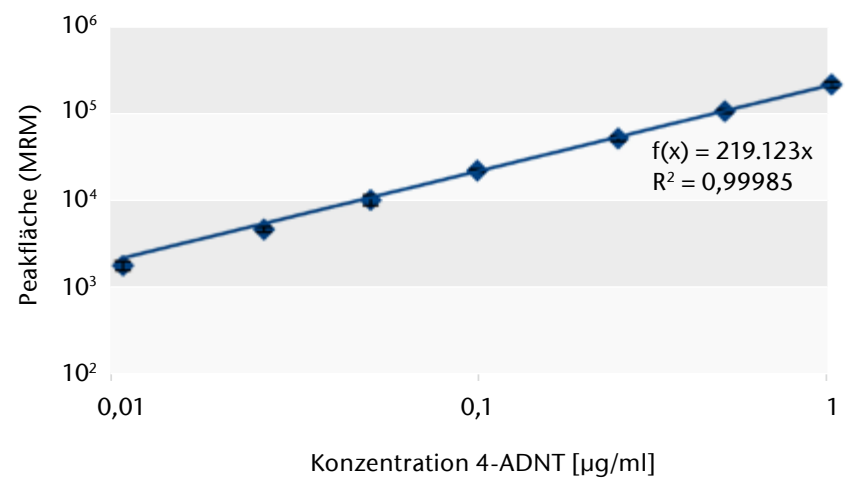

Abb. 3) Kalibrationsgeraden zur Bestimmung von 4-ADNT in Bodenproben ( $n=3)$; UV-Quantifizierung bei $254 \mathrm{~nm}(A)$ und MRM-Quantifizierung des spezifischen Fragments (hier: 196,1 > 119,15(-)) (B) in doppelt logarithmischer Darstellung.

Tab.3: Methodenvalidierung der UV- und MS-Quantifizierung hinsichtlich des linearen Bereichs, der unteren Nachweis(LOD) und Quantifizierungsgrenze (LOQ) sowie der Selektivität der chromatographischen Auftrennung zur nachfolgend eluierten STV (Peakauflösung $\mathrm{R}_{\mathrm{s}}$ )

\begin{tabular}{|c|c|c|c|c|c|}
\hline Methode & STV & $\begin{array}{l}\text { Linearer Bereich } \\
{[\mu \mathrm{g} / \mathrm{ml}]}\end{array}$ & $\begin{array}{l}\text { LOD } \\
{[\mu \mathrm{g} / \mathrm{ml}]}\end{array}$ & $\begin{array}{l}\mathrm{LOQ} \\
{[\mu \mathrm{g} / \mathrm{ml}]}\end{array}$ & Peakauflösung (UV) \\
\hline \multirow[t]{8}{*}{ UV } & TNB & $0,1-50$ & 0,25 & 0,50 & 5,52 \\
\hline & DNB & $0,1-50$ & 0,10 & 0,25 & 6,77 \\
\hline & TNT & $0,1-50$ & 0,25 & 0,50 & 1,13 \\
\hline & 4-ADNT & $0,1-50$ & 0,25 & 0,50 & 1,61 \\
\hline & 2-ADNT & $0,1-50$ & 0,25 & 0,50 & 0,98 \\
\hline & 2,4-DNT & $0,1-50$ & 0,10 & 0,25 & 0,96 \\
\hline & 2,6-DNT & $0,1-50$ & 0,25 & 0,50 & 3,90 \\
\hline & 2-NT & $0,1-50$ & 0,25 & 0,50 & - \\
\hline \multirow[t]{5}{*}{ MS } & TNB & $0,1-10$ & 0,10 & 0,25 & - \\
\hline & TNT & $0,1-25$ & 0,05 & 0,10 & - \\
\hline & 4-ADNT & $0,01-1$ & 0,01 & 0,025 & - \\
\hline & 2-ADNT & $0,01-1$ & 0,01 & 0,025 & - \\
\hline & 2,4-DNT & $0,1-10$ & 0,10 & 0,25 & - \\
\hline
\end{tabular}




\subsection{Optimierung der STV-Extraktion aus Bodenproben}

Aktuelle Referenzmethoden zur Extraktion von STV aus Bodenproben benötigen große Probenmengen von 10 bis $20 \mathrm{~g}$ und sind mit langwierigen Extraktionen bis zu $24 \mathrm{~h}$ verbunden (Environmental Protection Agency 2006; Deutsches Institut für Normung 2014). Um eine schnellere und trotzdem effektive Extraktion der hier betrachteten STV zu erreichen, wurden verschiedene Parameter optimiert. Zunächst wurde die Verringerung der eingesetzten Bodenmenge für zwei mit $1 \mathrm{~g} \mathrm{TNT/kg}$ dotierte Böden untersucht. Sowohl für den humushaltigen als auch für den sandigen Boden konnte kein großer Einfluss durch die Verringerung der eingesetzten Bodenmenge festgestellt werden (Abb. $4 \mathrm{~A}$ und B). Bei $1 \mathrm{~g}$ Boden fällt im Vergleich zu den anderen Proben lediglich die Standardabweichung höher aus. Für den sandigen Boden wurden etwas höhere Werte als $1 \mathrm{~g}$ TNT/kg Boden bestimmt. Beim humushaltigen Boden liegen die bestimmten Konzentrationen von ca. $700 \mathrm{mg} \mathrm{TNT} / \mathrm{kg}$ Boden deutlich unter der vormals dotierten Konzentration.

Durch diese Ergebnisse wurden $2 \mathrm{~g}$ Boden als ausreichend erachtet und in nachfolgenden Experimenten zur Ermittlung einer verkürzten Extraktionszeit eingesetzt. Für beide dotierte Böden wurden Extraktionszeiten von $1 \mathrm{~min}, 5 \mathrm{~min}, 10 \mathrm{~min}, 30 \mathrm{~min}, 60 \mathrm{~min}$ sowie $18 \mathrm{~h}$ als Referenzwert untersucht. Bereits nach 1 min konnten in allen Proben bis zu $75 \%$ der nach $18 \mathrm{~h}$ ermittelten TNT- , 2-ADNT- und 4-ADNT-Konzentrationen nachgewiesen werden (Abb. $4 \mathrm{C}$ und $\mathrm{D}$ ). Bis zur Extraktionszeit von 30 min kann eine weitere Zunahme festgestellt werden, die mit Ausnahme von 2-ADNT bis zur Maximalzeit von $18 \mathrm{~h}$ keine großen Veränderungen zeigt. Im TNT-dotierten Humusboden fielen die TNTKonzentrationen noch einmal niedriger als im Vorexperiment aus. Dafür konnten erhöhte Konzentrationen der Aminodinitrotoluole nachgewiesen werden, die im Sandboden nicht vorhanden waren.

Im letzten Optimierungsexperiment wurde überprüft, ob die STV-Extraktion aus dem dotierten Humusboden durch die Anwendung von Ultraschall verbessert werden kann. Nach 1 min Extraktionszeit wurden durch den UItraschall ca. 5-10\% mehr TNT extrahiert, allerdings gleicht sich die Konzentration der beiden Verfahren nach 5 min wieder an und fällt bei der einfachen Extraktion nach 30 min und 60 min sogar höher aus (Abb. 4 E). Nach $18 \mathrm{~h}$ zeigt sich wiederum ein leichter Vorteil des Ultraschallverfahrens.

Aufgrund der Ergebnisse zur Optimierung der Extraktion wurde ein verkürztes Verfahren etabliert, das für TNT und die Aminodinitrotoluole vergleichbare Ergebnisse zum Referenzverfahren lieferte. Dieses optimierte Verfahren nutzt eine geringe Bodenmenge ( $2 \mathrm{~g}$ ) und eine einfache Lösungsmittelextraktion mit Acetonitril bei einer deutlich verkürzten Extraktionszeit von $30 \mathrm{~min}$.

Abschließend wurden zur Validierung der gesamten Messmethodik inklusive des verkürzten Extraktionsverfahrens die Wiederfindungsrate und die Präzision bestimmt. Die Wiederfindungsrate wird zur Bewertung der Probenvorbereitung herangezogen. Hierfür wurde unbelasteter, humushaltiger Boden gezielt mit $10 \mu \mathrm{g} / \mathrm{ml}$ STV-Mix versetzt, im Anschluss mit dem optimierten Verfahren extrahiert und am LC-MS/MS analysiert. Mit Ausnahme von 2,4-DNT ist die Wiederfindungsrate der MS-Quantifizierung höher als bei der Verwendung der UV-Quantifizierung (Abb. 5 A). Alle anderen Wiederfindungsraten liegen mindestens bei $85 \%$, bei 2-ADNT sogar bei $95 \%$ (MS-Quantifizierung).

Zur Bestimmung der Präzision wurden für die dotierten Böden zehn Extraktionen durchgeführt und über LC-MS/MS quantifiziert. Die massenspektrometrische Quantifizierung lieferte etwas höhere TNT-Werte als der UV-Nachweis (Abb. 5 B). Die für die Präzision relevante Standardabweichung der TNT-Konzentrationen fällt bei dem dotierten Humusboden etwas höher als beim Sandboden aus. Die im Humusboden nachgewiesenen 2- und 4-ADNT zeigen dagegen nur eine geringe Standardabweichung.

Die optimierten Extraktions- und Messmethoden wurden an Realproben $(\mathrm{HO}-\mathrm{H} 4)$ getestet, die einige Wochen zuvor als Referenz von einem pro- fessionellen Anbieter (terracon $\mathrm{GmbH}$ Jüterbog) analysiert worden waren. In der Probe $\mathrm{HO}$ konnten mit beiden Verfahren keine STV nachgewiesen werden. Die Proben $\mathrm{H} 1$ bis $\mathrm{H} 4$ zeigen Kontaminationen mit TNT, 2-ADNT und 4-ADNT (Abb. 6). Im Vergleich zu den Referenzwerten sind unsere Messwerte niedriger. Insbesondere bei der hoch kontaminierten Probe $\mathrm{H} 1$ werden größere Abweichungen deutlich. Allerdings musste bei dieser Bodenprobe eine Probe der Dreifachbestimmung ausgeschlossen werden, da sie im Vergleich zu den anderen beiden Extrakten eine zehnfach erhöhte TNT-Konzentration aufwies. Die Quantifizierungsergebnisse der niedriger belasteten Proben liegen dagegen deutlich näher an den Referenzwerten.

\section{Diskussion}

\subsection{LC-MS/MS-Methodenentwicklung}

Die untersuchten Sprengstoffe unterscheiden sich in ihrer Polarität, was für die Separation in der Umkehrphasen-HPLC (RP-HPLC) genutzt werden kann. Eher polare Sprengstoffe, wie TNB oder DNB, eluieren zu Beginn des Gradienten, da der polare Anteil der mobilen Phase hier höher ist. Weniger polare Sprengstoffe wie 2-NT werden dagegen erst am Ende bei einem hohen organischen Anteil des Lösungsmittels eluiert. Mit der optimierten Methode konnten alle acht STV separiert werden. Aufgrund der sehr ähnlichen chemischen Struktur der Sprengstoffe zeigten sich im Chromatogramm teilweise Überlagerungen der Peaks. Dies wirkte sich negativ auf die Peakauflösung aus, so dass der Grenzwert von 1,5 teilweise unterschritten wurde. Insbesondere strukturelle Isomere wie 2- und 4-ADNT oder 2,4- und 2,6-DNT neigen zur Co-Elution in der Chromatographie (Bečanová et al. 2010), was sich negativ auf die Quantifizierung auswirken kann. Bei Erstellung der Kalibrationsgeraden und Quantifizierung der STV in Bodenproben wurde jedoch keine Beeinträchtigung beobachtet. Die Kalibration zeigte eine hohe Reproduzierbarkeit und ermittelte vergleichbare Ergebnisse zu der massenspektrometrischen Quantifizierung. 
Zum massenspektrometrischen Nachweis müssen die STV über ESI ionisierbar sein. Durch den Einsatz von Additiven wie Ammoniumacetat und Ammoniumhydroxid kann die Ionisation der Analyten deutlich verbessert werden (Kostiainen \& Kauppila 2009). So konnten TNT, 2-ADNT, 4-ADNT und 2,4-DNT als deprotonierte Anionen $[\mathrm{M}-\mathrm{H}]^{-}$nachgewiesen werden.
Von TNB wurde statt des deprotonierten Anions bereits ein spezifisches Fragment als Vorläufer-lon detektiert

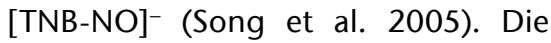
drei weiteren STV (DNB, 2,6-DNT, 2-NT) konnten aufgrund der geringen Anzahl von Nitrogruppen bzw. ihrer Struktur nicht über ESI ionisiert werden. Mithilfe der APCl (chemische Ionisation bei Atmosphärendruck) als alternative Ionisationsquelle wäre eine lonisation von DNB und 2,6-DNT möglich (Thurman \& Ferrer 2012). 2-NT kann dagegen nur über GC-MS (Gaschromatographie-Massenspektrometrie) nachgewiesen werden (Perr et al. 2005).

Für die ionisierbaren STV wurden im Zuge des MRM spezifische Fragmente (Produkt-Ionen) ermittelt und damit
A

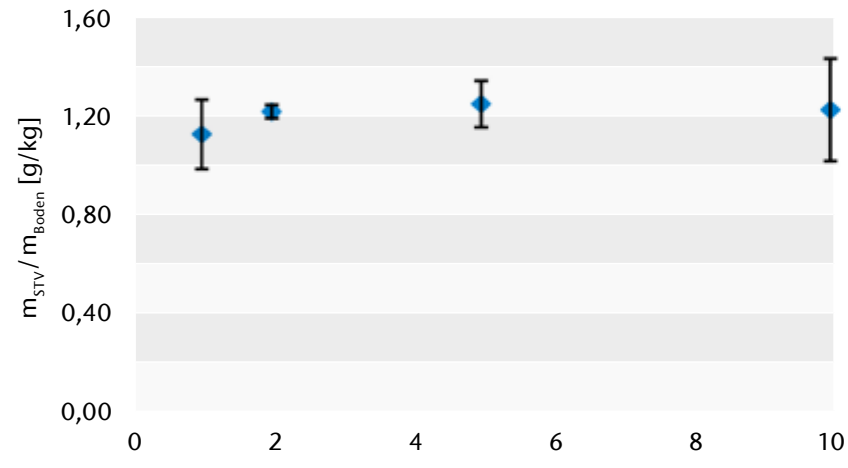

Bodenmenge [g]

\section{C}

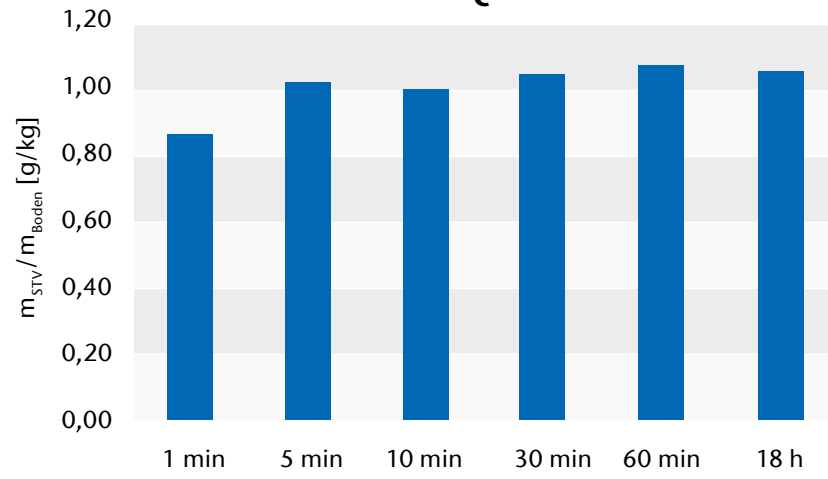

Extraktionszeit
B
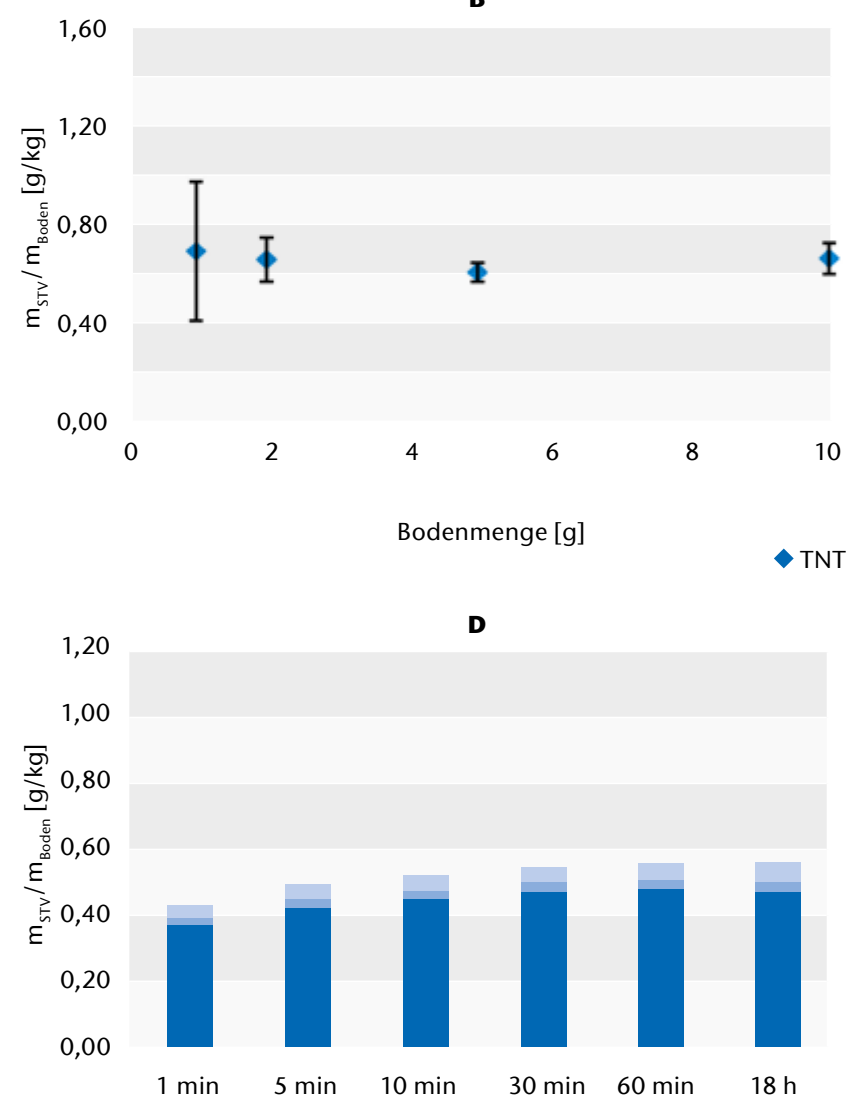

Extraktionszeit

$\because 4-\mathrm{ADNT}=2$-ADNT $\because \mathrm{TNT}$

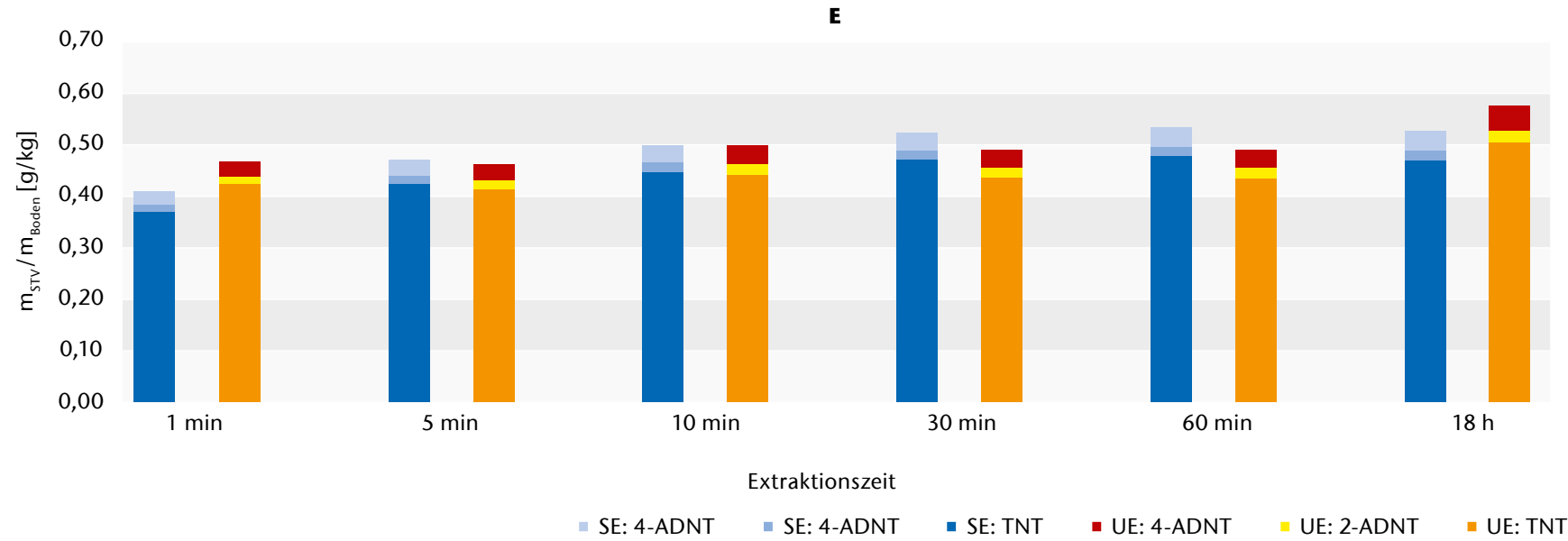

Abb. 4) Optimierung der STV-Extraktion aus dotierten Bodenproben: Nachgewiesene TNT-Konzentrationen bei Einsatz verschiedener Bodenmengen für sandigen (A) bzw. humushaltigen Boden (B); Einfluss der Extraktionsdauer auf die nachgewiesene STV-Konzentrationen in sandigem (C) und humushaltigem Boden (D); Vergleich der über Schüttel- (SE) und Ultraschallextraktion (UE) extrahierten STV in dotiertem Humusboden (E). 
A

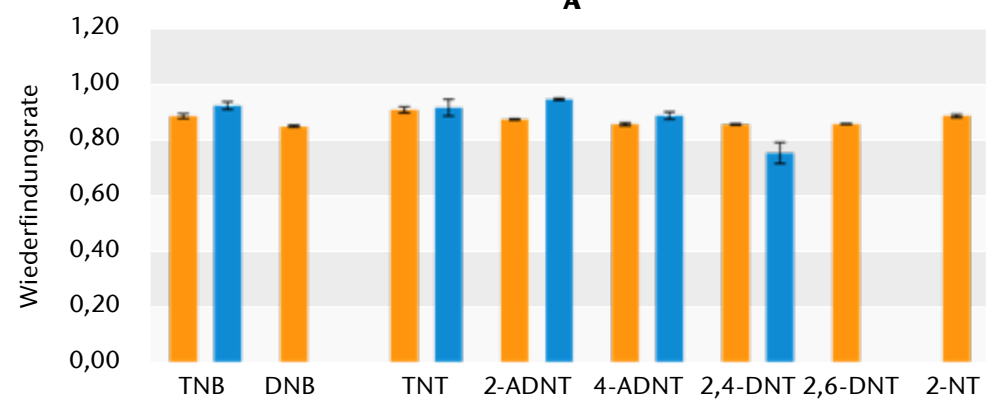

STV

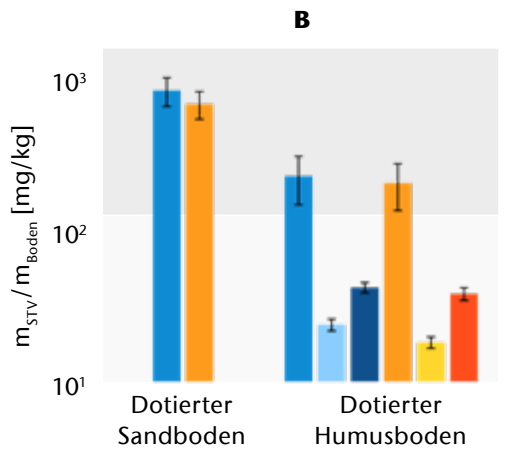

MS: TNT
MS: 2-ADNT
MS: 4-ADNT
- UV: TNT

UV: 2-ADNT

- UV: 4-ADNT

Abb. 5) Wiederfindungsrate von STV $(10 \mu \mathrm{g} / \mathrm{ml})$ in humushaltigen Bodenproben, die über die optimierten Methoden extrahiert und analysiert worden sind $(n=2)(A)$; Präzision der entwickelten Extraktions- und LC-MS/MS-Methoden für dotierten Sand- und Humusboden $(n=10)(B)$.

auch die Sensitivität für die Quantifizierung der STV deutlich erhöht. Selbst bei den Doppelpeaks der ausgewählten ADNT-Fragmente ist durch den jeweils spezifischen Zeitpunkt der Elution und die erhöhte Spezifität des Produkt-Ionen-Nachweises eine unabhängige Quantifizierung möglich. Bei der MS-Quantifizierung wurden für alle STV niedrigere LOD und LOQ im Vergleich zur UV-Quantifizierung ermittelt. Die Sprengstoffe mit der höchsten Sensitivität wiesen die niedrigste $\operatorname{LOD}(0,01 \mu \mathrm{g} / \mathrm{ml})$ auf, was im besseren lonisationsverhalten der ADNT-Isomere gegenüber TNT, TNB und 2,4-DNT begründet ist. Im Vergleich zur lonisationsmethode der APCI (LOD - 0,002 bis 0,027 $\mu \mathrm{g} / \mathrm{ml}$ ), die häufiger für den massenspektrometrischen STV-Nachweis eingesetzt wird, konnten für die hier untersuchten STV mit der ESI ähnlich gute LOD erzielt werden (Jiang 2010).

\subsection{STV-Extraktion aus Bodenproben}

Ein wichtiger Parameter bei der Durchführung einer Bodenextraktion ist die Probenvorbehandlung, bei der der Boden homogenisiert und bei Bedarf getrocknet wird. Boden ist eine komplexe Probenmatrix, und die Verteilung der STV in belasteten Gebieten ist meistens inhomogen (Landesamt für Umwelt, Gesundheit und Verbraucherschutz Brandenburg 2015). Dieses Verhalten konnte auch in den hier untersuchten Bodenproben beobachtet werden. So wurden im dotierten
Sandboden trotz einer 20-minütigen Homogenisation bis zu 10 \% höhere TNT-Konzentrationen bestimmt, als ursprünglich dotiert wurden. Diese Vermutung wird auch durch die im Validierungsprozess überprüfte $\mathrm{Me}$ thodenpräzision bestätigt, da größere Schwankungen der gemessenen TNTKonzentrationen in der UV- und MSBestimmung aufgetreten sind.

Beim dotierten Humusboden konnten noch andere Einflussfaktoren beobachtet werden. So wurde hier in allen Experimenten weniger TNT nachgewiesen, als dotiert wurde. Zudem nimmt die TNT-Konzentration von ca. $700 \mathrm{mg} / \mathrm{kg}$ Boden im ersten Experiment (Einfluss der eingesetzten Bodenmenge) auf ca. $250 \mathrm{mg} / \mathrm{kg}$ Boden im letzten Experiment zur Bewertung der Methodenpräzision ab. Aufgrund der Mehrfachbestimmung und der Untersuchung der weiteren Proben kann die Homogenisation hier nicht der einzige Grund für diese Abweichungen sein. Allerdings ist durch den hohen Humingehalt des Bodens ein erhöhtes Schadstoffrückhaltevermögen zu erwarten, d. h., es ist eine teilweise Bindung des TNT an Huminstoffe wahrscheinlich, das dann nicht mehr für Extraktionen zur Verfügung steht (Bayerisches Landesamt für Umwelt 2009). Zudem konnte neben der Abnahme der TNT-Konzentration eine Zunahme der ADNT in dem dotierten Humusboden festgestellt werden. So wurden im letzten Experiment reproduzierbar bis zu 100 mg ADNT/kg Boden nachgewiesen. Da sie nicht dem
Boden zugefügt worden waren, sind sie auf Transformationsprozesse im Boden zurückzuführen. Die Proben wurden dunkel, kühl und fest verschlossen gelagert, so dass photolytische Transformationsreaktionen unwahrscheinlich sind. Vielmehr könnten im Humusboden vorhandene Mikroorganismen das dotierte TNT zu 2-ADNT und 4-ADNT abgebaut haben. Ein weiteres Indiz hierfür ist die vermehrte Bildung von 4-ADNT gegenüber 2-ADNT, was für reduktive Mechanismen spricht (Monteil-Rivera et al. 2009).

Die Ultraschallextraktion konnte im Vergleich zur einfachen Extraktion nur eine geringfügige Erhöhung der extrahierten STV nach $18 \mathrm{~h}$ erzielen. Da das Ziel in der Entwicklung einer einfachen und schnellen Methode lag, die möglichst auch im Feld angewendet werden kann, ist daher die einfache Lösungsmittelextraktion vorzuziehen. In den hier untersuchten Bodenproben waren nur die häufigsten STV TNT, 2-ADNT und 4-ADNT vertreten. Für andere STV, wie z. B. TNB, müsste im Einzelnen geprüft werden, ob die verkürzte Extraktion angewendet werden kann oder andere Verfahren (z. B. Beschleunigte Lösungsmittelextraktion (ASE) oder Soxhlet-Extraktion) vorteilhafter sind.

Bei der Messung von Realproben wurden trotz geringer Standardabweichung deutliche Differenzen zu den vorliegenden Referenzwerten ermittelt. So fielen die meisten berechneten Konzentrationen niedriger aus und 


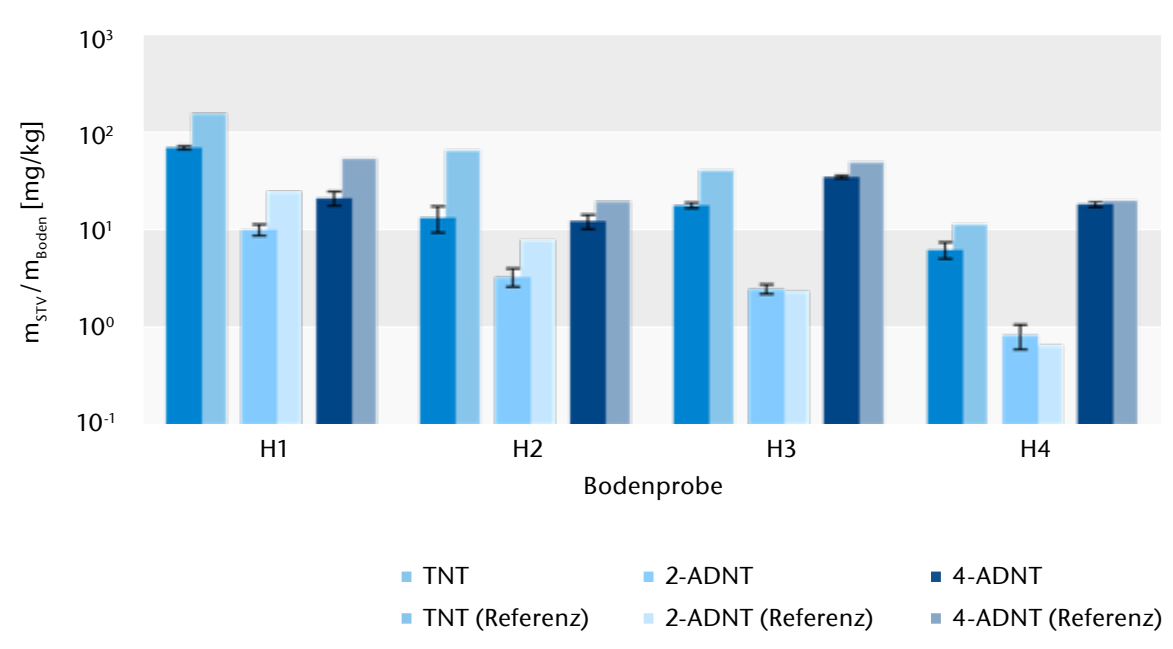

Abb. 6) LC-MS/MS-Bestimmung von TNT, 2-ADNT und 4-ADNT in kontaminierten Realproben mit den optimierten Methoden im Vergleich zu den Werten des Analytiklabors (terracon $\mathrm{GmbH}$ ) als Referenz (H1: $n=2 ; H 2-H 4: n=3)$.

lagen außerhalb des Vertrauensintervalls. Auch hier kommen zwei der bereits zuvor beobachteten Fakten zum Tragen. Zum einen lag vermutlich keine ausreichende Homogenisierung der entnommenen Gesamtprobe vor. Von den jeweils 10-20 kg entnommenen Bodenproben wurde nur ein sehr geringer Teil (insgesamt $6 \mathrm{~g}$ je Probe) in unserem Labor analysiert. Für die Probe $\mathrm{H} 1$ wurde in der Dreifachbestimmung trotz vorheriger Durchmischung sogar ein Wert mit zehnfach erhöhter TNT-Konzentration gegenüber den anderen beiden Extrakten ermittelt, der als Ausreißer nicht in die Auswertung einbezogen wurde. Eine unzureichende Durchmischung der Ausgangsmaterialien hat daher wahrscheinlich zu den großen Abweichungen zwischen unseren ermittelten Konzentrationen und den Werten des Referenzlabors geführt. Zum anderen lagen zwischen den Analysen mehrere Wochen, so dass insbesondere bei den huminhaltigen Proben (H2-H4) Transformations- und Bindungsprozesse stattgefunden haben könnten.

\section{Schlussfolgerung und Ausblick}

Die Laboranalyse sprengstofftypischer Verbindungen kann in drei größere Arbeitskomplexe unterteilt werden. Dazu zählen eine möglichst präzise Probenvorbereitung, die Extraktion der Sprengstoffe aus Bodenproben und die Analyse der Sprengstoffbelastung mittels geeigneter Quantifizierungsmethoden. Über die Proben-
Eine sensitivere und selektivere Detektion konnte durch die Etablierung einer MS-Quantifizierungsmethode für fünf der acht STV erreicht werden. Für TNB, TNT, 2-ADNT, 4-ADNT und 2,4DNT wurden geeignete Produkt- und Fragment-Ionen zum Quantifizieren ermittelt

Mit neuen und gut homogenisierten Realproben sollte die Anwendbarkeit der Methode noch einmal überprüft werden. Dafür wäre am besten ein Bodenmaterial geeignet, das auch Kontaminationen durch die anderen STV aufweist. Eine Alternative für kontaminierte Realproben wäre die Verwendung eines zertifizierten Referenzmaterials, bei dem die STV-Konzentrationen bekannt sind. So könnte der verkürzte Extraktionsvorgang auch für die anderen STV überprüft werden. In dieser Arbeit wurden nur die STV TNT, 4-ADNT und 2-ADNT nachgewiesen, die allerdings auch die häufigsten und bedeutendsten Kontaminationen auf Altlastenflächen darstellen. Trotzdem wäre es insbesondere für Nitramin-Sprengstoffe wie Oktogen (HMX) oder Hexogen (RDX) von Interesse, die Methode auf zusätzliche STV auszuweiten und auch Transformationsprodukte, wie z. B. Benzoesäuren, zu betrachten. Die Analyse eines breiteren Schadstoffspektrums setzt allerdings auch eine Neubewertung und eventuelle Verbesserung der chromatographischen Auftrennung voraus.

Schließlich konnte mit dieser Arbeit ein vereinfachtes und deutlich schnelleres Verfahren entwickelt werden, das für ein STV-Screening von Altlastenverdachtsflächen genutzt werden kann. Darauf aufbauend könnten an ausgewählten Kontaminationsherden Standardverfahren zur STV-Analyse eingesetzt werden, um eine ausreichende Gefährdungsabschätzung der betroffenen Liegenschaften zu treffen und Sanierungspläne zu erstellen. 


\section{LITERATUR}

Bayerisches Landesamt für Umwelt (2009) Arbeitshilfe für die Untersuchung von Sprengplätzen. Praxisteil, Augsburg

Bečanová J, Friedl Z, Šimek Z (2010) Identification and determination of trinitrotoluenes and their degradation products using liquid chromatography-electrospray ionization mass spectrometry. International Journal of Mass Spectrometry 291(3):133-139. doi: 10.1016/j. ijms.2010.01.016

DeArmond PD, DiGoregorio AL (2013) Characterization of liquid chromatography-tandem mass spectrometry method for the determination of acrylamide in complex environmental samples. Anal Bioanal Chem 405(12):4159-4166. doi: 10.1007/s00216-013-6822-4

Deutsches Institut für Normung (2014) Bodenbeschaffenheit - Bestimmung von ausgewählten Explosivstoffen und verwandten Verbindungen - Teil 2: Verfahren mittels Gaschromatographie (GC) und ElektronenEinfang-Detektion (ECD) oder massenspektrometrische Detektion (MS).(DIN-ISO-11916-2). Accessed 13 Nov 2017

Environmental Protection Agency (2006) Method$8330 \mathrm{~B}$. Nitroaromatics, nitramines, and nitrate esters by high performance liquid chromatography (HPLC).

Environmental Protection Agency (2017) Technical Fact Sheet - 2,4,6-Trinitrotoluene (TNT)

Herrmann B (2008) Untersuchungen der Transformationswege von 2,4,6 Trinitrotoluol in Oberflächengewässern und deren Nutzen als natürliche Schadstoffminderungsprozesse an einem ehemaligen Rüstungsstandort Dissertation, Philipps-Universität

liang G (2010) Simultaneous UHPLC/MS Analyses of Explosive Compounds. Thermo Scientific

Kostiainen R, Kauppila TJ (2009) Effect of eluent on the ionization process in liquid chromatography-mas spectrometry. I Chromatogr A 1216(4):685-699. doi: 10.1016/j.chroma.2008.08.095

Küchler F (2011) Probennahme und Qualitätssicherungsmaßnahmen bei Bodenkontaminationen mit sprengstofftypischen Verbindungen. Dissertation, Freie Universität Berlin

Landesamt für Umwelt, Gesundheit und Verbraucherschutz Brandenburg (2015) Arbeitshilfe Grundwasserkontaminationen mit sprengstofftypischen Verbindungen im Land Brandenburg. Behandlung, Aufnahmemechanismen, Abbauverhalten Stufe IB Umsetzung der Literaturrecherche und Erarbeitung vertiefender Grundlagen -. Fachinformation des LUGV. Altlastenbearbeitung im Land Brandenburg, Potsdam
Leito I, Kruve A, Rebane R, Oldekop M-L, Evard H, Herodes K, Kipper K, Helm I (2017) LC-MS Method Validation. Online Course. https://sisu.ut.ee/lcms_method_validation/course-introduction. Accessed 13 Nov 2017

Monteil-Rivera F, Halasz A, Groom C, Zhao I-S, Thiboutot S (2009) Fate and Transport of Explosives in the Environment. In: Sunahara GI, Lotufo G, Hawari J, Kuperman RG (eds) Ecotoxicology of explosives. Taylor \& Francis, Boca Raton, ISBN: 9781420004342, pp 5-33

Perr JM, Furton KG, Almirall JR (2005) Gas chromatography positive chemical ionization and tandem mass spectrometry for the analysis of organic high explosives. Talanta 67(2):430-436. doi: 10.1016/j. talanta.2005.01.035

Song Y, Chen H, Cooks RG (2005) Reactivity of acetony anion with nitroaromatics. An atmospheric pressure chemical ionization study. Rapid Commun. Mass Spectrom. 19(23):3493-3499. doi: 10.1002/rcm.2206

Thurman EM, Ferrer I (2012) Adapting EPA Method 8330B for Analysis of Explosives in Water to SPE and LC/ MS/MS. Application Note 5991-0676EN, Colorado

Via SM, Zinnert JC (2016) Impacts of explosive compounds on vegetation. A need for community scale investigations. Environ Pollut 208(Pt B):495-505. doi: 10.1016/j.envpol.2015.10.020

\section{AUTOREN}

Prof. Dr. Marcus Frohme

Liane Kober, M. Sc.

Molekulare Biotechnologie und Funktionelle Genomik Technische Hochschule Wildau

Christian Hanschke, M. Sc.

terracon - Laboratorium für Umwelt- und Pestizidanalytik $\mathrm{GmbH}$

Prof. Dr. Rainer Macholz Prof. Dr. Rainer Macholz Umweltprojekte $\mathrm{GmbH}$

E-Mail für Korrespondenz:

marcus.frohme@th-wildau.de

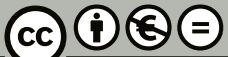

